\title{
Sistema computacional para índices de cárie dentária: banco de dados e análise estatística*
}

\section{A computer software system for dental caries rates: data bases and statistical analysis}

\author{
Maria Lucia M.M. Sundefeld e Sabina Léa Davidson Gotlieb \\ Departamento de Odontologia Social da Faculdade de Odontologia de Araçatuba da Universidade \\ Estadual Paulista. Araçatuba, SP - Brasil (M.L.M.M.S.), Departamento de Epidemiologia da Faculdade \\ de Saúde Pública da Universidade de São Paulo. São Paulo, SP - Brasil (S.L.D.G.)
}

\begin{abstract}
Resumo
Apresenta-se um sistema computacional, denominado ICADPLUS, desenvolvido para elaboração de banco de dados, tabulação de dados, cálculo do índice CPO e análise estatística para estimação de intervalos de confiança e comparação de resultados de duas populações. Tem como objetivo apresentar método simplificado para atender necessidades de serviços de saúde na área de odontologia processando fichas utilizadas por cirurgiões dentistas em levantamentos epidemiológicos de cárie dentária. A característica principal do sistema é a dispensa de profissional especializado na área de odontologia e computação, exigindo o conhecimento mínimo de digitação por parte do usuário, pois apresenta "menus" simples e claros como também relatórios padronizados, sem possibilidade de erro. Possui opções para fichas de CPO segundo Klein e Palmer, CPO proposto pela OMS, CPOS segundo Klein, Palmer e Knutson, e ceo. A validação do sistema foi feita por comparação com outros métodos, permitindo recomendar sua adoção.
\end{abstract}

Sistemas de informação, organização \& administração. Índice CPO.

\begin{abstract}
s
A computer software system developed ICADAPLUS, is presented in order to create and tabulate data bases, calculate the DMF rate, perform statistical comparison of two populations, and calculate confidence intervals. The system offers a simplified method for health services in the area of dentistry, using dental records to carry out epidemiological surveys of tooth decay. The system's main feature is that it does not require specialists either in the area of dentistry or computing, demanding of the user only basic data-entry typing skills, since it presents simple menus, and standardized reports, with no possibility of error. The system comprises four steps: Data-entry, Processing, Reports and Utilities. In Data-entry the regions, towns and institutions supplying the data are initially registered, once only. Each record receives a
\end{abstract}

\footnotetext{
* Baseado na dissertação de mestrado, apresentada à Faculdade de Saúde Pública da Universidade de São Paulo, 1995.

Correspondência para/Correspondence to: Maria Lucia M.M. Sundefeld - Departamento de Odontologia Social da Faculdade de Odontologia de Araçatuba da Universidade do Estado de São Paulo. Rua José Bonifácio, 1193 - 16015-050 Araçatuba, SP - Brasil. Fax: (018) 623.2638 E-mail: mlsundef@infocenter.com.br

Edição subvencionada pela FAPESP. Processo 95/2290-6.

Recebido em 30.8.1995. Reapresentado em 15.2.1996. Aprovado em 21.3.1996.
} 
code number, and it is this code which is available to the user through a Function Key, by means of which the code is transferred to the desired data field. Furthermore, there is a choice of type of dental chart: Klein and Palmer's $D M F$; the DMF proposed by the WHO; Klein, Palmer and Knutson's DMF surfaces; or Gruebbel's def. It is possible to include, alter and exclude records in each option. In Processing, it is necessary, first of all, to organise the indexes, totalize the data, and remit them to the data base, where no further alteration or exclusion occurs. From this stage on, reports can be prepared from the proposed indexes, grouped according to age, or age and gender. The system was validated by comparison with other methods. This comparison justifies the recomendation of its implementation.

Information systems, organization and administration. DMF index.

\section{INTRODUÇÃO}

Segundo Bertrand" " "pesquisador, envolvido em coleta populacional de dados de saúde, freqüentemente se defronta com grande número de dificuldades associadas a pesquisas tipo surveys". Esse citado autor apresenta fatores que dificultam esse trabalho, tais como, definição do tamanho da amostra, instrumento de avaliação de resultados, planos de análises inapropriados, estudos de alto custo e, se envolverem mais de uma cidade ou país, os problemas estarão multiplicados.

Segundo Anker ${ }^{2}$, há necessidade de introdução de novos métodos de avaliação reunindo técnicas epidemiológicas, estatísticas e antropológicas que forneçam informações rapidamente, a baixo custo e de forma simples. Entretanto, métodos rápidos de avaliação não podem ser usados indiscriminadamente e descuidadamente apenas por serem rápidos e muito pouco dispendiosos. Muitos desses métodos não têm ainda fundamentação científica e raramente foram sujeitos à revisão rigorosa antes de serem utilizados, e necessitam ser avaliados. O desenvolvimento da informática, com o surgimento dos microcomputadores, veio facilitar o desempenho do pesquisador nesses tipos de estudos.

Segundo Adams e col. ${ }^{1}$ e Bertrand ${ }^{5}$, o desenvolvimento de sistemas computacionais aplicados à saúde tem sido amplamente reconhecido como pesquisa prioritária, embora ainda haja dificuldades na implantação desses sistemas.

Newbrun ${ }^{16}$ salienta a importância do computador em odontologia, não somente na pesquisa, mas tam- bém em nível clínico, pois seria usado em hospitais ou universidades e também em consultórios particulares, no diagnóstico do risco da cárie.

Segundo Preston ${ }^{20}$, na década passada houve pouco envolvimento de computadores para facilitar a prática de dentistas. Com isto, os cirurgiões dentistas adotaram com maior frequiência sistemas computadorizados administrativos. Stheeman ${ }^{32}$ verificou que entre $1984 \mathrm{e}$ 1991 foram publicados 608 artigos apresentando sistemas computacionais em revistas de medicina e somente dois em periódicos de odontologia.

Hoje em dia, já existem alguns programas computacionais descritos na literatura ${ }^{5,9,10,11,14,21,34}$, sendo de aplicação na odontologia relacionados à concentração de flúor na saliva, detecção automática de lesões cariogênicas em imagens obtidas por filmes radiográficos, diagnóstico radiográfico diferencial, atribuição de escores em lesões de dentes molares, arquivos de pacientes com campos relacionados a problemas odontológicos e concentração de fluoreto na saliva.

Segundo Seixas ${ }^{31}$, as informações disponíveis sobre sistema odontológico, em nível mundial, são extremamente escassas ou pouco compatíveis entre si, pelo fato do quadro epidemiológico ser essencialmente dinâmico; se não for oportunamente descrito, no período que acontece, perde-se um material que é precioso no sentido de fornecer informações sobre um estágio de evolução sistêmica em odontologia.

Ao mesmo tempo, Chaves ${ }^{7}$ Newbrun ${ }^{16}$ enfatizam que sendo a cárie dentária um dos problemas de saúde pública, é necessário o seu controle através de levantamentos periódicos em populações. 
A Organização Mundial da Saúde ${ }^{18}$ preconiza que "levantamentos para determinar o estado de saúde bucal e as necessidades de tratamento de comunidades e populações são parte essencial das atribuições dos dirigentes e de outros administradores responsáveis pelo serviço odontológico". Isto porque, a cárie dentária e a doença periodontal existem em todas as populações, variando apenas em intensidade e prevalência, e apresentam alta percentagem da população afetada.

Vários estudos têm sido desenvolvidos no sentido de procurar causas de ocorrência da cárie dentária e tudo indica que o processo de evolução da mesma é específico de cada raça e cada população ${ }^{6,15,17}$.

Levantamentos epidemiológicos de cárie dentária vêm sendo realizados com freqüência, orientados por universidades ou por serviços de saúde bucal de diferentes partes do País ${ }^{3,23,24,25, ~ 26, ~ 27, ~ 28, ~ 29, ~} 30$.

Da mesma forma, o grupo de trabalho da Rede CEDROS $^{22}$ (Rede para Cooperação em Estudos e Desenvolvimento de Recursos Odontológicos para o Setor Saúde) tem abordado questões fundamentais para a organização e implementação das ações de saúde bucal nos diversos municípios brasileiros, e considera que "o diagnóstico da situação de saúde bucal,da área geográfica para a qual se está planejando o sistema de saúde, é essencial para a definição da política de saúde bucal". Salientam a necessidade de indicadores epidemiológicos de agravos à saúde e, não existindo dados referentes, faz-se necessária a realização de levantamentos epidemiológicos para que se possam dimensionar esses agravos, possibilitando o planejamento das ações necessárias para reduzi-los e controlá-los.

Entretanto, a grande dificuldade das autoridades responsáveis pela saúde bucal é o processamento dos dados coletados pelos seus profissionais. Os levantamentos são realizados e as fichas ficam guardadas, acumulando-se, ano a ano, isto porque, os serviços de saúde têm falta de pessoal treinado para trabalhar com dados coletados por cirurgiões dentistas, havendo pois necessidade de um programa que venha oferecer um instrumento de organização, tabulação e análise dos mesmos, sem a necessidade de pessoal qualificado na área de odontologia e computação.

Freqüentemente são realizados levantamentos de cárie dentária para determinação do índice $\mathrm{CPO}^{12,13}$ representando a história anterior e atual da cárie dentária. Como história anterior, são considerados os dentes obturados e extraídos e, como história atual, os dentes cariados, sendo que estes, dependendo do estágio avançado da cárie, poderão ser considerados extrações indicadas. O índice descreve numericamente os resultados, em média, do ataque de cárie aos dentes permanentes em um grupo populacional ${ }^{7}$. Este índice constitui o objeto de estudo do presente trabalho.

O índice "ceo", expresso pelo número médio de dentes decíduos que compõem o índice, por criança, bem como o CPO continuam sendo utilizados até agora, embora em 1986, a Organização Mundial da Saúde ${ }^{18}$ tenha proposto novos parâmetros para os mesmos para coleta de informações básicas sobre o estado de doenças bucais e necessidades de tratamento para o planejamento e controle dos programas de saúde bucal. Assim, para o cálculo do índice CPO, e para elaborar relatórios nos moldes dos já existentes, alguns códigos da OMS são automaticamente agrupados e substituídos pelo seu correspondente de Klein e Palmer ${ }^{12}$.

As combinações da condição dental e tratamentos e o seu correspondente para o CPO de Klein e Palmer estão apresentadas nas Tabelas 1 e 2.

Tabela 1 - Códigos para condição dental, indicação de tratamento para os dentes permanentes e correspondentes Klein e Palmer.

\begin{tabular}{ccc}
\hline \multicolumn{3}{c}{ Dentes permanentes } \\
\hline Cond. dental & Tratamento & Correspondente K\&P \\
\hline 8 & 0 ou 8 & "0" \\
1 ou 2 & 2,3 ou 6 & $" 1 "$ \\
3 & 0 ou 1 & $" 2 "$ \\
4 ou 5 & qualquer & $" 3 "$ \\
1 ou 2 & 7 & $" 4 "$ \\
0 & 0 ou 1 & $" 5 "$ \\
6 ou 7 & qualquer & $" 5 "$ \\
\hline
\end{tabular}

Tabela 2 - Códigos para condição dental, indicação de tratamento para os dentes decíduos e correspondentes Klein e Palmer.

\begin{tabular}{ccc}
\hline \multicolumn{3}{c}{ Dentes decíduos } \\
\hline Cond. dental & Tratamento & Correspondente K\&P \\
\hline B e C & 2,3 ou 6 & $" 6 "$ \\
B e C & 7 & $" 8 "$ \\
D & 0 ou 1 & $" 7 "$ \\
E & & \\
A, F ou G & & "9" \\
\hline
\end{tabular}

Considerando a necessidade de instrumentos para facilitar e agilizar diagnósticos de condições de saúde da população, propôs-se produzir tecnologia na área da informática, aplicada à saúde pública, elaborando um sistema simples, no idioma português, que facilitará o processamento de dados coletados em levantamentos referentes à cárie dentária, determinando prevalência de cárie com rapidez e precisão. Técnico de nível médio, com alguma prática de digitação em microcomputador, poderá utilizar o sistema através de telas simples e claras de entrada de dados. 
O presente trabalho tem como objetivo construir um instrumento auxiliar ao diagnóstico de saúde bucal, via microcomputador, relacionado à cárie dentária, de fácil utilização em odontologia social, que permita fazer estimativas de prevalência e comparações estatísticas entre resultados de inquéritos epidemiológicos de localidades diferentes ou entre resultados de épocas diferentes em uma mesma localidade.

\section{ESTRUTURA DO SISTEMA PROPOSTO}

O sistema desenvolvido, denominado ICADPLUS, tem como significado abranger mais que o cálculo do Índice de Cárie Dentária, elaborando análises estatísticas sobre os dados armazenados no banco de dados construído pelo próprio sistema, desenvolvido em linguagem Clipper 5.01, podendo ser usado tanto por mono ${ }^{33}$ como por multiusuário $^{19}$. $\mathrm{O}$ banco de dados com capacidade ilimitada para guardar dados coletados relativos a comunidades ou municípios, podendo estes ser agrupados por regiões geográficas, dentro de um ou mais países.

O sistema dá possibilidade de utilização em rede e o equipamento mínimo necessário é microcomputador da linha PC-AT 286 ou superior, com monitor (monocromático ou colorido), possuindo no mínimo $640 \mathrm{~Kb}$ de memória RAM, com Winchester de 30 mgbytes ou mais de capacidade, dependendo do volume de dados de interesse da instituição usuária, sendo que 1,8 mgbytes serão ocupados pelo sistema.

As estatísticas geradas pelo sistema foram as seguintes: média atimética; desvio padrão; proporção de cada componente dentro do CPO; estimação por intervalo de confiança de $95 \%$ para médias populacionais; comparação entre dados médios.

Considerando que levantamentos epidemiológicos de cárie dentária deverão abranger número grande de pessoas, e que a Organização Mundial de Saúde ${ }^{18}$ sugere entre 40 e 50 indivíduos o tamanho da amostra por faixa etária, quando os valores CPO para a idade de 12 anos variam de moderados para muito altos, no sistema ICADPLUS o número mínimo foi limitado em 60 , para que, com amostras suficientemente grandes, as distribuições dos índices CPO e ceo estudados possam ser consideradas como normais.

A percentagem da diferença entre duas médias é definida sempre considerando a média da primeira população como $100 \%$, e em função dela calcula-se o valor percentual desta diferença. O teste para comparação de duas médias é o aplicado a duas populações distintas, não correlacionadas. Ao se realizar levantamento epidemiológico para determinação do índice de cárie dentária, CPO, estar-se-á sempre trabalhando com populações não correlacionadas, uma vez que o levantamento epidemiológico é um estudo transversal, realizado em um determinado momento, onde cada pessoa examinada não será comparada com ela mesma em nenhuma situação. Mesmo que esta pessoa faça parte de um novo levantamento em época diferente, ela entrará em uma nova faixa etária que não coincidirá com a anterior. $\mathrm{O}$ sistema analisa o valor encontrado de $z$, com decisões em nível de significância de $5 \%$.

No caso de não haver o mínimo exigido para o tamanho da amostra, o teste não será realizado, dando a mensagem "tamanho insuficiente", o que não impede que, com os valores das médias e desvios-padrão calculados pelo sistema e apresentados nos relatórios oferecidos pelo mesmo, o usuário calcule a estatística " $\mathrm{t}$ " de STUDENT ${ }^{4,8}$

\section{DESCRIÇÃO DAS TELAS E RELATÓRIOS DO SISTEMA}

Ao iniciar a execução do sistema, aparecerá a primeira das vinte e uma telas de "menu", com uma apresentação simples para o fácil entendimento e operação do mesmo, e, a partir desta tela, surgirão as subsequientes, tais como:

\section{Tela de Inclusão/Alteração e Exclusão de Registros}

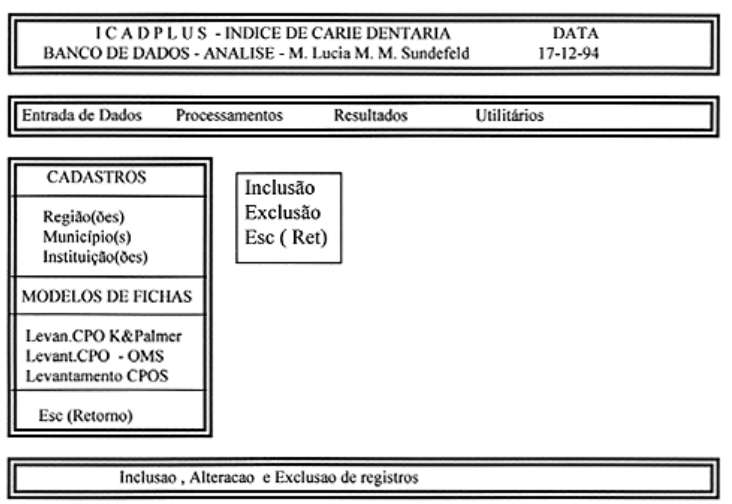

O primeiro passo para o uso do ICADPLUS é o cadastramento das regiões, municípios e instituições, levando a barra luminosa sobre cada uma das opções. Cada região será cadastrada somente uma vez, assim como cada município e instituição, onde serão sempre representados por um código numérico, não havendo mais, a partir daí, necessidade de repetir a digitação dos seus nomes.

As fichas só serão digitadas depois de feito os cadastramentos das localidades onde forem realizados os levantamentos, tanto região, como município, como instituição.

$\mathrm{Na}$ tela de cadastramento da região constam dados do censo populacional; na tela município constam dados relativos ao total de habitantes, tipo de economia, número de unidades odontológicas e o código da região a que pertence; na tela instituição, constam variáveis de identificação, como, nome, en- 
dereço, área de localização, tipo de estabelecimento, número de unidades odontológicas e número de pessoas filiadas a mesma. Se a instituição for escola, haverá possibilidade de registrar os níveis educacionais, ficando também vinculada aos códigos do município e região a que pertence.

\section{Ficha para CPOD e ceo, segundo Klein e Palmer}

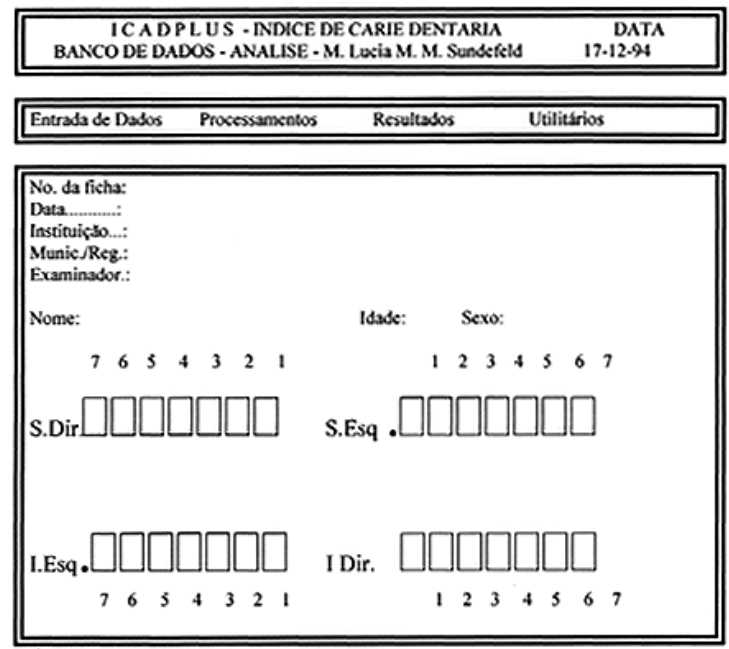

As telas de entrada de dados para cálculo dos índices propostos obedecerão aos critérios e fichas definidos pelos profissionais da área de odontologia e utilizados nos levantamentos.

As telas correspondentes aos três tipos de fichas são as seguintes:

Ficha para CPOD e ceo proposta pela OMS

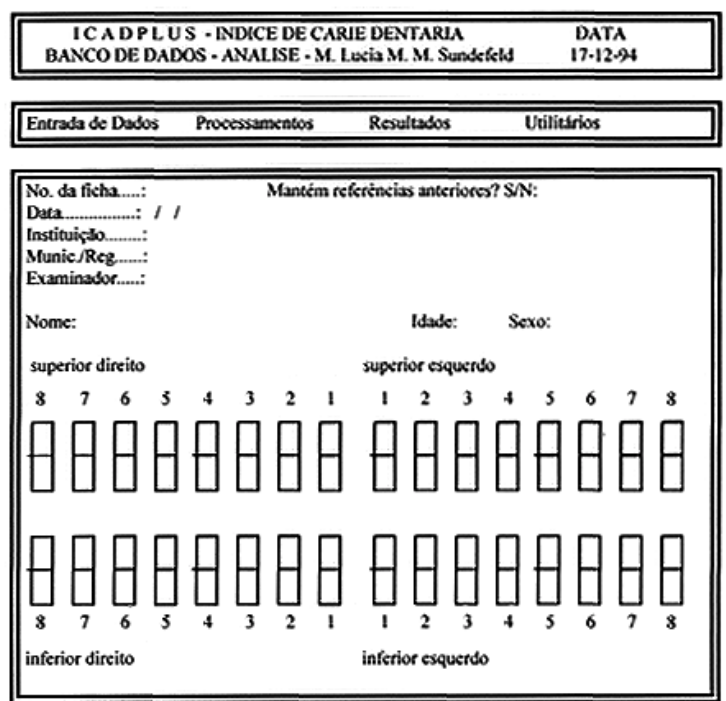

A ficha seguinte necessita de duas telas, pois para CPOS são necessários cinco campos para cada dente, tomando mais espaço para a digitação. Por isso, foram separados os arcos, superior e inferior.

Ficha para CPOS segundo Klein, Palmer e Knutson

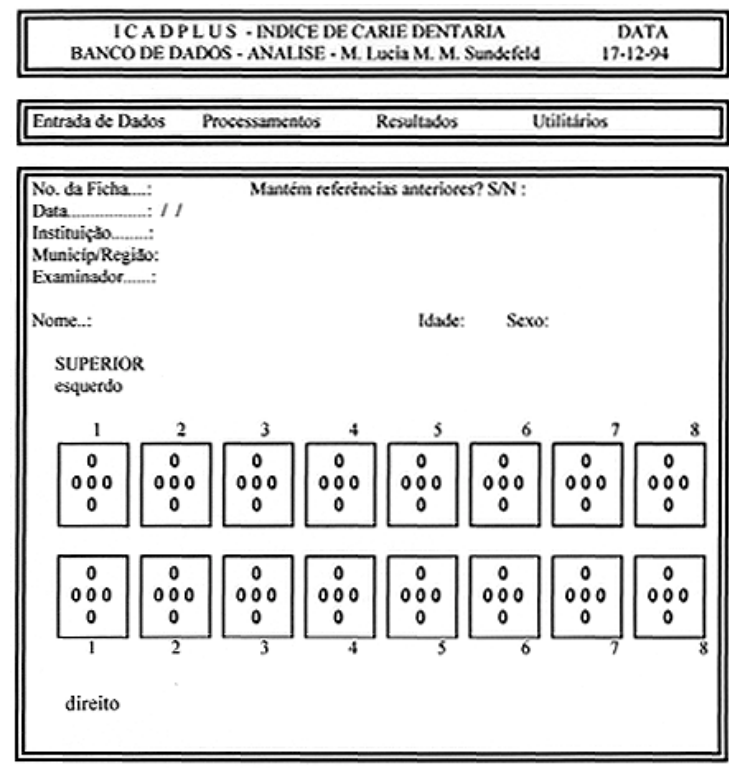


Terminada a digitação do arco superior, a tela rola automaticamente, aparecendo nova tela para o arco inferior, onde todo cabeçalho já estará preenchido.

$\mathrm{Na}$ fase de processamento de dados serão gerados todos os índices nos moldes necessários para que todos os procedimentos programados sejam possíveis.

Em seguida, é feita, via "menu", a apuração e sumarização dos dados, processando a tabulação dos dados separadamente tanto para fichas de Klein e Palmer, para dente ou superfície, como para fichas da OMS.

Ao terminar a digitação e conferência dos dados, os mesmos serão transferidos para um outro arquivo, produzindo o banco de dados, onde não mais serão feitas alterações, deixando o arquivo de entrada de dados disponível para digitação de novo levantamento, iniciando novamente a numeração das fichas.

A seguir, passa-se para a confecção e emissão dos relatórios,os quais foram programados para apresentar o CPOD, CPOS e ceo, tanto para unidade dente como para superfície, nos moldes tradicionais, tais como:

Relatório Individual: Apresenta a contagem dos códigos de cada pessoa examinada, agrupando-as por idade e sexo. Cada linha do relatório corresponde aos códigos de uma pessoa tanto dos dentes permanentes como dos decíduos. Manter-se-á a mesma apresentação quando a unidade analisada for superfície do dente.

\section{Relatório Individual}

Período:

Instituição:

Município:

Região:

Idade:

Sexo:

\begin{tabular}{ccccccccc}
\hline$n^{\circ}$ ficha & $n^{\circ}$ exames & $c$ & $o$ & $e$ & $e i$ & $c p o$ & $t i$ & $h$ \\
\hline $1+i$ & $l$ & $x$ & $y$ & $z$ & $w$ & $x+y+z+w$ & $k$ & $h$ \\
\hline
\end{tabular}

\begin{tabular}{lcclcccc}
\hline$n^{\circ}$ ficha & $n^{\circ}$ exames & $c$ & $o$ & $e$ & $c e o$ & $t p$ & $h$ \\
\hline $1+i$ & $l$ & $x$ & $y$ & $w$ & $x+y+w$ & $p$ & $h$ \\
\hline
\end{tabular}

para: i variando de 0 a r sendo $1+r$ total de pessoas examinadas, onde,

$\mathrm{x}=$ total de dentes ou superfícies cáriados por pessoa $(\mathrm{C} / \mathrm{c})$

$\mathrm{y}=$ total de dentes ou superfícies obturados por pessoa $(\mathrm{O} / \mathrm{o})$

$\mathrm{Z}=$ total de dentes ou superfícies extraídos por pessoa $(\mathrm{E})$

$\mathrm{w}=$ total de dentes ou superfícies com extrações indicadas por pessoa (Ei / e)

$\mathrm{k}=$ total de dentes ou superfícies irrompidos $(\mathrm{TI})$

$\mathrm{p}=$ total de dentes temporários presentes (tp)

$\mathrm{h}=$ total de dentes ou superfícies hígidos $(\mathrm{H} / \mathrm{h})$

Tabela Geral: Cada linha corresponde à contagem total de cada código nos grupos de pessoas examinadas em cada idade e sexo . Existe a opção para se obter, em um mesmo relatório, tabela para dentes permanentes e tabela para dentes decíduos, ou relatório somente dos dentes permanentes ou somente dos dentes decíduos, tanto para unidade dente como para unidade superfície. Isto porque nem sempre são examinadas pessoas em idade de possuir dentes permanentes e decíduos ao mesmo tempo, ou por não haver interesse por parte do usuário em obter relatório conjunto de permanentes e decíduos. 


\section{Relatório Geral dos Índices}

\begin{tabular}{cccccccccc}
\hline Idade & Sexo & No Ex. & c & o & e & ei & cpo & ti & h \\
\hline \multirow{4}{*}{$\mathrm{I}+\mathrm{i}$} & $\mathrm{j}_{1}$ & $\mathrm{n}_{1}$ & $\mathrm{x}_{1}$ & $\mathrm{y}_{1}$ & $\mathrm{z}_{1}$ & $\mathrm{w}_{1}$ & $\mathrm{x}_{1}+\mathrm{y}_{1}+\mathrm{z}_{1}+\mathrm{w}_{1}$ & $\mathrm{k}_{1}$ & $\mathrm{~h}_{1}$ \\
& $\mathrm{j}_{2}$ & $\mathrm{n}_{2}$ & $\mathrm{x}_{2}$ & $\mathrm{y}_{2}$ & $\mathrm{z}_{2}$ & $\mathrm{w}_{2}$ & $\mathrm{x}_{2}+\mathrm{y}_{2}+\mathrm{z}_{2}+\mathrm{w}_{2}$ & $\mathrm{k}_{2}$ & $\mathrm{~h}_{2}$ \\
& $\mathrm{j}_{\mathrm{t}}$ & $\mathrm{n}_{\mathrm{t}}$ & $\mathrm{x}_{\mathrm{t}}$ & $\mathrm{y}_{\mathrm{t}}$ & $\mathrm{z}_{\mathrm{t}}$ & $\mathrm{w}_{\mathrm{t}}$ & $\mathrm{x}_{\mathrm{t}}+\mathrm{y}_{\mathrm{t}}+\mathrm{z}_{\mathrm{t}}+\mathrm{w}_{\mathrm{t}}$ & $\mathrm{k}_{\mathrm{t}}$ & $\mathrm{h}_{\mathrm{t}}$ \\
\hline
\end{tabular}

\begin{tabular}{ccccccccc}
\hline Idade & Sexo & No Ex. $^{\circ}$ & $\mathrm{c}$ & $\mathrm{o}$ & $\mathrm{e}$ & ceo & $\mathrm{tp}$ & $\mathrm{h}$ \\
\hline \multirow{2}{*}{$\mathrm{I}+\mathrm{i}$} & $\mathrm{j}_{1}$ & $\mathrm{n}_{1}$ & $\mathrm{x}_{1}$ & $\mathrm{y}_{1}$ & $\mathrm{w}_{1}$ & $\mathrm{x}_{1}+\mathrm{y}_{1}+\mathrm{w}_{1}$ & $\mathrm{p}_{1}$ & $\mathrm{~h}_{1}$ \\
& $\mathrm{j}_{2}$ & $\mathrm{n}_{2}$ & $\mathrm{x}_{2}$ & $\mathrm{y}_{2}$ & $\mathrm{w}_{2}$ & $\mathrm{x}_{2}+\mathrm{y}_{2}+\mathrm{w}_{2}$ & $\mathrm{p}_{2}$ & $\mathrm{~h}_{2}$ \\
& $\mathrm{j}_{\mathrm{t}}$ & $\mathrm{n}_{\mathrm{t}}$ & $\mathrm{x}_{\mathrm{t}}$ & $\mathrm{y}_{\mathrm{t}}$ & $\mathrm{w}_{\mathrm{t}}$ & $\mathrm{x}_{\mathrm{t}}+\mathrm{y}_{\mathrm{t}}+\mathrm{w}_{\mathrm{t}}$ & $\mathrm{p}_{\mathrm{t}}$ & $\mathrm{h}_{\mathrm{t}}$ \\
\hline
\end{tabular}

onde,

$\mathrm{I}=$ menor idade do levantamento

i variando de 0 a $r$, sendo $I+r=$ maior idade do levantamento.

os índices $(1,2, \mathrm{t})$ representam:

$1=$ feminino, $2=$ masculino e $\mathrm{t}=$ ambos os sexos

$\mathrm{xj}=$ total de dentes ou superfícies cariados por idade e sexo $(\mathrm{C} / \mathrm{c})$

$\mathrm{yj}=$ total de dentes ou superfícies obturados por idade e sexo $(\mathrm{O} / \mathrm{o})$

$\mathrm{Z}_{\mathrm{j}}=$ total de dentes ou superfícies extraídas por idade e sexo ( E )

$\mathrm{wj}=$ total de dentes ou superfícies com extrações indicadas por idade e sexo (Ei/e)

$\mathrm{xt}=$ total de dentes ou superfícies cariados por idade

$\mathrm{y}_{\mathrm{t}}=$ total de dentes ou superfícies obturados por idade

$\mathrm{z}_{\mathrm{t}}=$ total de dentes ou superfícies extraídos por idade

$\mathrm{w}_{\mathrm{t}}=$ total de dentes ou superfícies com extrações indicadas, por idade

$\mathrm{k}_{\mathrm{j}}=$ total de dentes ou superfícies irrompidos por idade e sexo (TI)

$\mathrm{p}_{\mathrm{j}}=$ total de dentes ou superfícies temporários presentes (tp)

$h_{\mathrm{j}}=$ total de dentes ou superfícies hígidas por idade e sexo $(\mathrm{H} / \mathrm{h})$

$\mathrm{k}_{\mathrm{t}}=$ total de dentes ou superfícies irrompidos por idade

$\mathrm{p}_{\mathrm{t}}=$ total de dentes ou superfícies temporários presentes por idade

$\mathrm{h}_{\mathrm{t}}=$ total de dentes ou superfícies hígidas por idade

$\mathrm{n}_{\mathrm{j}}=$ total de pessoas examinadas de cada sexo de uma mesma idade

Índice Médio: Corresponde ao índice CPO propriamente dito para cada idade e sexo, com base nos agrupamentos feitos no relatório geral, existindo também a opção de um relatório onde apresenta CPO e ceo juntos, tanto para unidade dente como para unidade superfície, ou relatórios separados somente para o índice CPO ou para o índice ceo, também podendo ser para unidade dente ou superfície.

\section{Relatório do Índice Médio}

\begin{tabular}{ccccccccccc}
\hline Idade & Sexo & No Ex. & $\mathrm{c}$ & $\mathrm{o}$ & $\mathrm{e}$ & ei & cpo & d.p. & ti & $\mathrm{h}$ \\
\hline \multirow{3}{*}{$\mathrm{I}+\mathrm{i}$} & $\mathrm{j}_{1}$ & $\mathrm{n}_{1}$ & $\mathrm{x}_{1} / \mathrm{n}_{1}$ & $\mathrm{y}_{1} / \mathrm{n}_{1}$ & $\mathrm{z}_{1} / \mathrm{n}_{1}$ & $\mathrm{w}_{1} / \mathrm{n}_{1}$ & $\left(\mathrm{x}_{1}+\mathrm{y}_{1}+\mathrm{z}_{1}+\mathrm{w}_{1}\right) / \mathrm{n}_{1}$ & $\mathrm{~s}_{1}$ & $\mathrm{k}_{1}$ & $\mathrm{~h}_{1}$ \\
& $\mathrm{j}_{2}$ & $\mathrm{n}_{2}$ & $\mathrm{x}_{2} / \mathrm{n}_{2}$ & $\mathrm{y}_{2} / \mathrm{n}_{2}$ & $\mathrm{z}_{2} / \mathrm{n}_{2}$ & $\mathrm{w}_{2} / \mathrm{n}_{2}$ & $\left(\mathrm{x}_{2}+\mathrm{y}_{2}+\mathrm{z}_{2}+\mathrm{w}_{2}\right) / \mathrm{n}_{2}$ & $\mathrm{~s}_{2}$ & $\mathrm{k}_{2}$ & $\mathrm{~h}_{2}$ \\
& $\mathrm{j}_{\mathrm{t}}$ & $\mathrm{n}_{\mathrm{t}}$ & $\mathrm{x}_{\mathrm{t}} / \mathrm{n}_{\mathrm{t}}$ & $\mathrm{y}_{\mathrm{t}} / \mathrm{n}_{\mathrm{t}}$ & $\mathrm{z}_{\mathrm{t}} / \mathrm{n}_{\mathrm{t}}$ & $\mathrm{w}_{\mathrm{t}} / \mathrm{n}_{\mathrm{t}}$ & $\left(\mathrm{x}_{\mathrm{t}}+\mathrm{y}_{\mathrm{t}}+\mathrm{z}_{\mathrm{t}}+\mathrm{w}_{\mathrm{t}}\right) / \mathrm{n}_{\mathrm{t}}$ & $\mathrm{s}_{\mathrm{t}}$ & $\mathrm{k}_{\mathrm{t}}$ & $\mathrm{h}_{\mathrm{t}}$ \\
\hline
\end{tabular}

\begin{tabular}{cccccccccc}
\hline Idade & Sexo & No Ex. & $\mathrm{c}$ & $\mathrm{o}$ & $\mathrm{e}$ & ceo & d.p. & tp & $\mathrm{h}$ \\
\hline \multirow{3}{*}{$\mathrm{I}+\mathrm{i}$} & $\mathrm{j}_{1}$ & $\mathrm{n}_{1}$ & $\mathrm{x}_{1} / \mathrm{n}_{1}$ & $\mathrm{y}_{1} / \mathrm{n}_{1}$ & $\mathrm{w}_{1} / \mathrm{n}_{1}$ & $\left(\mathrm{x}_{1}+\mathrm{y}_{1}+\mathrm{w}_{1}\right) / \mathrm{n}_{1}$ & $\mathrm{~s}_{1}$ & $\mathrm{p}_{1}$ & $\mathrm{~h}_{1}$ \\
& $\mathrm{j}_{2}$ & $\mathrm{n}_{2}$ & $\mathrm{x}_{2} / \mathrm{n}_{2}$ & $\mathrm{y}_{2} / \mathrm{n}_{2}$ & $\mathrm{w}_{2} / \mathrm{n}_{2}$ & $\left(\mathrm{x}_{2}+\mathrm{y}_{2}+\mathrm{w}_{2}\right) / \mathrm{n}_{2}$ & $\mathrm{~s}_{2}$ & $\mathrm{p}_{2}$ & $\mathrm{~h}_{2}$ \\
& $\mathrm{j}_{\mathrm{t}}$ & $\mathrm{n}_{1}+\mathrm{n}_{2}$ & $\mathrm{x}_{\mathrm{t}} / \mathrm{n}_{\mathrm{t}}$ & $\mathrm{yt} / \mathrm{n}_{\mathrm{t}}$ & $\mathrm{wt} / \mathrm{n}_{\mathrm{t}}$ & $\left(\mathrm{xt}+\mathrm{yt}+\mathrm{w}_{\mathrm{t}}\right) / \mathrm{n}_{\mathrm{t}}$ & $\mathrm{s}_{\mathrm{t}}$ & $\mathrm{p}_{\mathrm{t}}$ & $\mathrm{h}_{\mathrm{t}}$ \\
\hline
\end{tabular}


onde,

$\mathrm{I}=$ menor idade do levantamento

i variando de 0 a r , sendo $\mathrm{I}+\mathrm{r}=$ maior idade do levantamento.

os índices $(1,2, \mathrm{t})$ representam:

$1=$ feminino, $2=$ masculino $\mathrm{e} t=$ ambos os sexos

$\mathrm{xj}=$ total de dentes ou superfícies cariados por idade e sexo $(\mathrm{C} / \mathrm{c})$

$\mathrm{yj}=$ total de dentes ou superfícies obturados por idade sexo $(\mathrm{O} / \mathrm{o})$

$\mathrm{Z}_{\mathrm{j}}=$ total de dentes ou superfícies extraídos por idade e sexo ( E )

$\mathrm{w}_{\mathrm{j}}=$ total de dentes ou superfícies com extrações indicadas por idade e sexo (Ei /e)

$s j=$ desvio-padrão por idade e sexo

$\mathrm{k}_{\mathrm{j}}=$ total de dentes ou superfícies irrompidos por idade e sexo

$\mathrm{p}_{\mathrm{j}}=$ total de dentes ou superfícies temporários presentes por idade e sexo

$\mathrm{h}_{\mathrm{j}}=$ total de dentes ou superfícies hígidos por idade e sexo

$\mathrm{x}_{\mathrm{t}}=$ total de dentes ou superfícies cariados por idade

$\mathrm{y}_{\mathrm{t}}=$ total de dentes ou superfícies obturados por idade

$\mathrm{z}_{\mathrm{t}}=$ total de dentes ou superfícies extraídos por idade

$\mathrm{w}_{\mathrm{t}}=$ total de dentes ou superfícies com extrações indicadas, por idade

$\mathrm{s}_{\mathrm{t}}=$ desvio-padrão de uma mesma idade

$\mathrm{k}_{\mathrm{t}}=$ total de dentes ou superfícies irrompidos em uma mesma idade

$\mathrm{p}_{\mathrm{t}}=$ total de dentes ou superfícies temporários presentes em uma mesma idade

$\mathrm{h}_{\mathrm{t}}=$ total de dentes ou superfícies hígidos em uma mesma idade

$\mathrm{n}_{\mathrm{j}}=$ total de pessoas de cada sexo e de uma mesma idade

$\mathrm{n}_{\mathrm{t}}=$ total de exames em cada idade

Composição Percentual dos Índices: Refere-se às observações para cada idade e sexo, também baseado nos agrupamentos do relatório geral, com as mesmas opções dos anteriores.

\section{Relatório da Composição Percentual dos Índices}

\begin{tabular}{cccccccc}
\hline Idade & Sexo & No exames & $\mathrm{c}$ & $\mathrm{o}$ & $\mathrm{e}$ & $\mathrm{ei}$ & $\mathrm{cpo}$ \\
\hline \multirow{3}{*}{$\mathrm{I}+\mathrm{i}$} & $\mathrm{j}_{1}$ & $\mathrm{n}_{1}$ & $\mathrm{x}_{1} \%$ & $\mathrm{y}_{1} \%$ & $\mathrm{z}_{1} \%$ & $\mathrm{w}_{1} \%$ & $100 \%$ \\
& $\mathrm{j}_{2}$ & $\mathrm{n}_{2}$ & $\mathrm{x}_{2} \%$ & $\mathrm{y}_{2} \%$ & $\mathrm{z}_{2} \%$ & $\mathrm{w}_{2+} \%$ & $100 \%$ \\
& $\mathrm{j}_{\mathrm{t}}$ & $\mathrm{n}_{\mathrm{t}}$ & $\mathrm{x}_{\mathrm{t}} \%$ & $\mathrm{y}_{\mathrm{t}} \%$ & $\mathrm{z}_{\mathrm{t}} \%$ & $\mathrm{w}_{\mathrm{t}} \%$ & $100 \%$ \\
\hline
\end{tabular}

\begin{tabular}{ccccccc}
\hline Idade & Sexo & Noexames & $\mathrm{c}$ & $\mathrm{o}$ & $\mathrm{e}$ & ceo \\
\hline \multirow{3}{*}{$\mathrm{I}+\mathrm{i}$} & $\mathrm{j}_{1}$ & $\mathrm{n}_{1}$ & $\mathrm{x}_{1} \%$ & $\mathrm{y}_{1} \%$ & $\mathrm{w}_{1} \%$ & $100 \%$ \\
& $\mathrm{j}_{2}$ & $\mathrm{n}_{2}$ & $\mathrm{x}_{2} \%$ & $\mathrm{y}_{2} \%$ & $\mathrm{w}_{2} \%$ & $100 \%$ \\
& $\mathrm{j}_{\mathrm{t}}$ & $\mathrm{n}_{\mathrm{t}}$ & $\mathrm{x}_{\mathrm{t}} \%$ & $\mathrm{y}_{\mathrm{t}} \%$ & $\mathrm{w}_{\mathrm{t}} \%$ & $100 \%$ \\
\hline
\end{tabular}

onde,

$\mathrm{I}=$ menor idade do levantamento

i variando de 0 a $r$, sendo $\mathrm{I}+\mathrm{r}=$ maior idade do levantamento.

os índices $(1,2, \mathrm{t})$ representam:

$1=$ feminino, $2=$ masculino $\mathrm{e} t=$ ambos os sexos

xj $\%=$ porcentagem de dentes ou superfícies cariados por idade e sexo em relação ao respectivo $\mathrm{CPO}$ ou ceo

yj $\%=$ porcentagem de dentes ou superfícies obturados por idade e sexo em relação ao respectivo $\mathrm{CPO}$ ou ceo

zj \% = porcentagem de dentes ou superfícies extraídos por idade e sexo em relação ao respectivo $\mathrm{CPO}$ 
wj $\%=$ porcentagem de dentes ou superfícies com extrações indicadas por idade e sexo em relação ao respectivo $\mathrm{CPO}$ ou ceo

xt $\%=$ porcentagem dos dentes ou superfícies cariados em uma mesma idade em relação ao respectivo $\mathrm{CPO}$ ou ceo

yt $\%=$ porcentagem de dentes ou superfícies obturados em uma mesma idade em relação ao respectivo $\mathrm{CPO}$ ou ceo

zt $\%=$ porcentagem de dentes ou superfícies extraídos em uma mesma idade em relação ao respectivo $\mathrm{CPO}$ ou ceo

wt $\%=$ porcentagem de dentes ou superfícies com extração indicada em em uma mesma idade em relação ao respectivo $\mathrm{CPO}$ ou ceo

nj = número total de exames em cada sexo e de uma mesma idade

$\mathrm{n}_{\mathrm{t}}=$ número total de exames em uma mesma idade

Relatórios das Fichas Preconizadas pela OMS: Estes relatórios apresentam a contagem dos códigos, representando as condições dos dentes, utilizados nas fichas preconizadas pela OMS, tanto para dentes permanentes, como para dentes decíduos.

\section{Relatório das Condições Dentais segundo a OMS}

\begin{tabular}{|c|c|c|c|c|c|c|c|c|c|c|c|}
\hline \multicolumn{12}{|c|}{ Dentes permanentes } \\
\hline Idade & Sexo & № Ex. & Hígido & Cariado & Rest/c & Rest. & Per/c & Perd. & Selan & ApoiP. & Não Irr \\
\hline \multirow{3}{*}{$\mathrm{I}+\mathrm{i}$} & $\mathrm{j}_{1}$ & $\mathrm{n}_{1}$ & $\mathrm{~h}_{1}$ & $\mathrm{x}_{1}$ & $a_{1}$ & $\mathrm{y}_{1}$ & $\mathrm{~b}_{1}$ & $e_{1}$ & $\mathrm{c}_{1}$ & $d_{1}$ & $\mathrm{k}_{1}$ \\
\hline & $\mathrm{j}_{2}$ & $\mathrm{n}_{2}$ & $\mathrm{~h}_{2}$ & $\mathrm{x}_{2}$ & $\mathrm{a}_{2}$ & $\mathrm{y}_{2}$ & $b_{2}$ & $\mathrm{e}_{2}$ & $c_{2}$ & $\mathrm{~d}_{2}$ & $\mathrm{k}_{2}$ \\
\hline & $\mathrm{j}_{\mathrm{t}}$ & $n_{t}$ & $h_{t}$ & $x_{t}$ & $a_{t}$ & $y_{t}$ & $b_{t}$ & $e_{t}$ & $c_{t}$ & $d_{t}$ & $\mathrm{k}_{\mathrm{t}}$ \\
\hline Idade & Sexo & № Ex. & Hígido & Cariado & Rest/c & Rest. & Per/c & Selar & \multicolumn{2}{|c|}{ ApoiP. } & \\
\hline \multirow{3}{*}{$\mathrm{I}+\mathrm{i}$} & $\mathrm{j}_{1}$ & $\mathrm{n}_{1}$ & $\mathrm{~h}_{1}$ & $\mathrm{x}_{1}$ & $\mathrm{a}_{1}$ & $\mathrm{y}_{1}$ & $\mathrm{~b}_{1}$ & $\mathrm{~d}_{1}$ & \multicolumn{2}{|c|}{$\mathrm{k}_{1}$} & \\
\hline & $\mathrm{j}_{2}$ & $\mathrm{n}_{2}$ & $\mathrm{~h}_{2}$ & $\mathrm{x}_{2}$ & $\mathrm{a}_{2}$ & $\mathrm{y}_{2}$ & $\mathrm{~b}_{2}$ & $\mathrm{~d}_{2}$ & \multicolumn{2}{|c|}{$\mathrm{k}_{2}$} & \\
\hline & $\mathrm{j}_{\mathrm{t}}$ & $n_{t}$ & $h_{t}$ & $x_{t}$ & $a_{t}$ & $y_{t}$ & $b_{t}$ & $d_{t}$ & \multicolumn{2}{|c|}{$\mathrm{k}_{\mathrm{t}}^{2}$} & \\
\hline
\end{tabular}

onde,

$\mathrm{I}=$ menor idade do levantamento

i variando de 0 a $r$, sendo $I+r=$ maior idade do levantamento.

os índices $(1,2, \mathrm{t})$ representam:

$1=$ feminino, $2=$ masculino e $\mathrm{t}=$ ambos os sexos

$\mathrm{h}_{\mathrm{j}}=$ total de dentes hígidos por idade e sexo

$\mathrm{x}_{\mathrm{j}}=$ total de dentes cariados por idade e sexo

$\mathrm{a}_{\mathrm{j}}=$ total de dentes restaurados com cárie por idade e sexo

$\mathrm{y}_{\mathrm{j}}=$ total de dentes restaurados por idade e sexo

$b_{j}=$ total de dentes perdidos por cárie

$e_{j}=$ total de dentes perdidos por outras razões

$\mathrm{c}_{\mathrm{j}}=$ total de dentes com selante ou verniz

$\mathrm{d}_{\mathrm{j}}=$ total de dentes como apoio de ponte ou coroa

$\mathrm{k}_{\mathrm{j}}^{\mathrm{j}}=$ total de dentes não irrompidos

$\mathrm{n}_{\mathrm{j}}=$ total de exames em cada sexo e de uma mesma idade

$\mathrm{n}_{\mathrm{t}}=\mathrm{n}_{1}+\mathrm{n}_{2}$ número total de dentes em uma mesma idade

$h_{t}, x_{t}, a_{t}, y_{t}, b_{t}, e_{t}, c_{t}, d_{t}, k_{t}=$ totais das condições para ambos os sexos. 


\section{Relatórios de Tratamentos Indicados para Dentes Permanentes ou Decíduos}

\begin{tabular}{cccccccccccc}
\hline \multicolumn{10}{c}{ Dentes permanentes e decíduos } \\
\hline Idade & Sexo & $N^{0}$ Ex. & Nenh. & c.car/sel & obt1s & obt2s & coroa & elem.p. & trat.p & estr. & outros \\
\hline \multirow{3}{*}{$+\mathrm{i}$} & $\mathrm{j}_{1}$ & $\mathrm{n}_{1}$ & $\mathrm{l}_{1}$ & $\mathrm{~m}_{1}$ & $\mathrm{p}_{1}$ & $\mathrm{q}_{1}$ & $\mathrm{r}_{1}$ & $\mathrm{~s}_{1}$ & $\mathrm{u}_{1}$ & $\mathrm{v}_{1}$ & $\mathrm{~d}_{1}$ \\
& $\mathrm{j}_{2}$ & $\mathrm{n}_{2}$ & $\mathrm{l}_{2}$ & $\mathrm{~m}_{2}$ & $\mathrm{p}_{2}$ & $\mathrm{q}_{2}$ & $\mathrm{r}_{2}$ & $\mathrm{~s}_{2}$ & $\mathrm{u}_{2}$ & $\mathrm{v}_{2}$ & $\mathrm{~d}_{2}$ \\
& $\mathrm{j}_{\mathrm{t}}$ & $\mathrm{n}_{\mathrm{t}}$ & $\mathrm{l}_{\mathrm{t}}$ & $\mathrm{m}_{\mathrm{t}}$ & $\mathrm{p}_{\mathrm{t}}$ & $\mathrm{q}_{\mathrm{t}}$ & $\mathrm{r}_{\mathrm{t}}$ & $\mathrm{s}_{\mathrm{t}}$ & $\mathrm{u}_{\mathrm{t}}$ & $\mathrm{v}_{\mathrm{t}}$ & $\mathrm{d}_{\mathrm{t}}$ \\
\hline
\end{tabular}

sendo, tanto para dentes permanentes como para decíduos, onde:

$\mathrm{I}=$ menor idade do levantamento

i variando de 0 a r , sendo $\mathrm{I}+\mathrm{r}=$ maior idade do levantamento

os índices $(1,2, \mathrm{t})$ representam:

$1=$ feminino, $2=$ masculino e $\mathrm{t}=$ ambos os sexos

$1_{\mathrm{j}}=$ nenhum tratamento indicado

$\mathrm{m}_{\mathrm{j}}=$ controle de cárie ou selante

$\mathrm{p}_{\mathrm{j}}=$ obturação de uma superfície

$\mathrm{q}_{\mathrm{j}}=$ obturação de duas ou mais superfícies

$\mathrm{r}_{\mathrm{j}}=$ coroa ou apoio de ponte

$\mathrm{s}_{\mathrm{j}}=$ elemento de ponte

$\mathrm{u}_{\mathrm{j}}=$ tratamento pulpar

$\mathrm{v}_{\mathrm{j}}=$ extração

$\mathrm{d}_{\mathrm{j}}^{\mathrm{j}}=$ outros cuidados

$\mathrm{l}_{\mathrm{t}}, \mathrm{m}_{\mathrm{t}}, \mathrm{p}_{\mathrm{t}}, \mathrm{q}_{\mathrm{t}}, \mathrm{r}_{\mathrm{t}}, \mathrm{s}_{\mathrm{t}}, \mathrm{u}_{\mathrm{t}}, \mathrm{v}_{\mathrm{t}}, \mathrm{d}_{\mathrm{t}}$ correspondem aos tratamentos indicados para ambos os sexos.

Para os relatórios seguintes, a seqüência estatística foi programada para trabalhar não somente com idade por idade, mas dando liberdade de montagem de grupos etários de acordo com o interesse do usuário e com a disponibilidade do número de pessoas examinadas em cada idade. Isto porque, no agrupamento por faixa etária, o número de pessoas examinadas será a soma dos examinados em todas as idades da faixa escolhida, permitindo um número necessário para a análise estatística proposta.

Embora não tenham sido fixados os agrupamentos por idade, são sugeridos na tela os preconizados pela OMS, tais como: 35 a 44 anos, 65 a 74 anos, e, considerando que, no Brasil, 60 anos já se inclui na classificação de idoso, aconselha-se também a formação de mais um grupo de 60 a 64 anos. As idades restantes também constarão nos relatórios, uma a uma.

Há a possibilidade de fazer a estimação por intervalo de confiança e teste de médias de duas populações, permitindo os seguintes relatórios.

\section{Relatório por Intervalo de Confiança (95\%) para CPO}

\begin{tabular}{cccccc}
\hline G.Etár & $\mathrm{N}^{\mathrm{o}}$.Ex & Sexo & CPOmédio & D.Padrão & Interv.de Confiança(95\%) \\
\multirow{3}{*}{ Ii -Is } & $\mathrm{n}_{1}$ & $\mathrm{j}_{1}$ & $\mathrm{X}_{1}$ & $\mathrm{dp}_{1}$ & $\mathrm{Li}_{1}$ \\
& $\mathrm{n}_{2}$ & $\mathrm{j}_{2}$ & $\mathrm{X}_{2}$ & $\mathrm{dp}_{2}$ & $\mathrm{Li}_{2}$ \\
& $\mathrm{nt}$ & $\mathrm{j}_{\mathrm{t}}$ & $\mathrm{X}_{\mathrm{t}}$ & $\mathrm{dp}_{\mathrm{t}}$ & $\mathrm{Li}_{\mathrm{t}}$ \\
\hline
\end{tabular}

onde,

$\mathrm{Ii}=$ Limite inferior do grupo etário

Is = Limite superior do grupo etário

os índices $(1,2, \mathrm{t})$ representam:

$1=$ feminino, $2=$ masculino $\mathrm{e} t=$ ambos os sexos

$I_{i}-I_{s}=$ faixa etária ( intervalos fechados ) 
$\mathrm{X}_{\mathrm{j}}=\mathrm{CPO}$ médio para cada sexo de uma faixa etária

$\mathrm{X}_{\mathrm{t}}=\mathrm{CPO}$ médio de ambos os sexos de uma faixa etária

$\mathrm{dp}_{\mathrm{j}}=$ desvio-padrão do CPO, para cada sexo de uma faixa etária

$\mathrm{dp}_{\mathrm{t}}=$ desvio-padrão do CPO de ambos os sexos de uma faixa etária

$\mathrm{Li}_{1}, \mathrm{Li}_{2}=$ limites inferiores dos intervalos de confiança para cada sexo em uma

faixa etária

$\mathrm{Ls}_{1}, \mathrm{Ls}_{2}=$ limites superiores dos intervalos de confiança para cada sexo em

uma faixa etária

$\mathrm{Li}_{\mathrm{t}}=$ limite inferior de cada faixa etária para ambos os sexos

$\mathrm{Ls}_{\mathrm{t}}=$ limite superior de cada faixa etária para ambos os sexos

$\mathrm{n}_{\mathrm{t}}=$ total de exames por faixa etária

Do mesmo modo, o relatório por intervalo de confiança para o índice ceo obedece o mesmo modelo do apresentado para o índice CPO.

Teste de Médias de Duas Populações

\begin{tabular}{lcccccccc}
\hline G.Etár & Sexo & N.1 $^{\circ}$ & N.2 & $\begin{array}{c}\text { Dif.entre } \\
\text { médias }\end{array}$ & \% dif & Erro P. & Estat.t & Decisão(5\%) \\
\hline \multirow{2}{*}{ Ii -Is } & $\mathrm{j}_{1}$ & $\mathrm{n}_{1 \mathrm{j}}$ & $\mathrm{n}_{2 \mathrm{j}}$ & $\mathrm{X}_{1 \mathrm{j}}-\mathrm{X}_{2 \mathrm{j}}$ & $\left(\mathrm{X}_{1 \mathrm{j}}-\mathrm{X}_{2 \mathrm{j}}\right) \%$ & epdif & $\mathrm{z}_{1}$ & diferem/não dif. \\
& $\mathrm{j}_{2}$ & $\mathrm{n}_{1 \mathrm{j}}$ & $\mathrm{n}_{2 \mathrm{j}}$ & $\mathrm{X}_{1 \mathrm{j}}-\mathrm{X}_{2 \mathrm{j}}$ & $\left(\mathrm{X}_{1 \mathrm{j}}-\mathrm{X}_{2 \mathrm{j}}\right) \%$ & epdif & $\mathrm{z}_{2}$ & diferem/não dif. \\
& $\mathrm{j}_{\mathrm{t}}$ & $\mathrm{n}_{1 \mathrm{t}}$ & $\mathrm{n}_{2 \mathrm{t}}$ & $\mathrm{X}_{1 \mathrm{~T}}-\mathrm{X}_{2 \mathrm{~T}}$ & $\left(\mathrm{X}_{1 \mathrm{t}}-\mathrm{X}_{2 \mathrm{t}}\right) \%$ & epdif & $\mathrm{z}_{\mathrm{t}}$ & diferem/não dif. \\
\hline
\end{tabular}

onde,

li = Limite inferior do grupo etário

ls = Limite superior do grupo etário

os índices $(1,2, \mathrm{t})$ representam:

$1=$ feminino, 2 = masculino e $\mathrm{t}=$ ambos os sexos

$\mathrm{I}_{\mathrm{i}}-\mathrm{I}_{\mathrm{s}}=$ faixa etária (intervalos fechados)

$\mathrm{X}_{\mathrm{j}}=\mathrm{CPO}$ médio para cada sexo de uma faixa etária

$\mathrm{X}_{\mathrm{t}}=\mathrm{CPO}$ médio de ambos os sexos de uma faixa etária

epdif $=$ erro padrão da diferença

$\mathrm{z}_{\mathrm{j}}=$ estatística do teste para cada sexo de uma faixa etária

$\mathrm{z}_{\mathrm{t}}=$ estatística do teste de ambos os sexos de uma faixa etária

\section{COMENTÁRIOS}

O sistema construído para cálculo e análise do índice CPO constituiu-se num sistema prático, de fácil utilização dispensando profissional especializado para manipulação dos dados coletados em levantamentos epidemiológicos de cárie dentária.

O banco de dados específico para cárie dentária mostrou-se eficiente e preciso. O sistema dois testado a partir do armazenamento de aproximadamente 16.000 fichas, onde cada levantamento pode ser localizado através do nome da região, município ou instituição, e da data de sua realização.
A validação do sistema implicou em um teste formal, quando foram observados os resultados obtidos por procedimentos manuais e por meio de relatórios emitidos por outro sistema processado em paralelo, o Epi Info, versão 6*. Posteriormente, estes foram comparados com os resultados obtidos pelo sistema proposto, tendo-se encontrado concordância perfeita.

O sistema desenvolvido poderá abranger todos os outros índices relacionados a saúde bucal, uma vez que suas estruturas foram planejadas prevendo a possibilidade de sua expansão.

* Sistema desenvolvido pelo "Center for Disease Control and Prevention”, Atlanta, Georgia, USA, 1994, em colaboração com a Organização Mundial de Saúde. 


\section{REFERÊNCIAS BIBLIOGRÁFICAS}

1. ADAMS, I. D. et al. Computer-aided diagnosis of abdominal pain: a multi-center study. Br. Med. J., 293: 800-4, 1986.

2. ANKER, M. Epidemiological and statistical methods for rapid health assessment: introduction. World Health Stat. Q., 44: 94-7, 1991.

3. ARCIERI, R. M. Avaliação da redução na incidência da cárie dentária, devido a bochechos com solução de fluoreto de sódio a 0,2\%. Rev. Assoc. Paul. Cir. Dent., 35:126-31, 1981.

4. BERQUÓ, E. S. et al. Bioestatística. São Paulo, E.P.U., 1981.

5. BERTRAND, W. E. Microcomputer applications in health population surveys: experience and potential in developing countries. World Health Stat. Q., 38: 91-100, 1985.

6. BURNETT, A. V. \& SCHERP, H. W. Oral microbiology infectious disease. 7th. ed. Baltimore, Williams \& Wilkins, 1968.

7. CHAVES, M. Odontologia social. 3a. ed. São Paulo, Artes Médicas, 1986.

8. COSTA NETO, P. L. O. Estatística. São Paulo, Edgard Blucher,1989.

9. GHIRLANDA, C. et al. New software for a prevention program in orodental pathology. Prev.Assist.Dent., 16: 4-9,1990.

10. HEAVEN.T. J. et al. Computer-based image analysis of natural approximal caries on radiographic films. J.Dent.Res., 71: 846-9, 1992.

11. JOSHI, A. Improving dental epidemiologic data collection with computer. J. Public Health Dent., 52: 232-8, 1992.

12. KLEIN, H. \& PALMER,C. E. Dental caries in American indian children. Public Health Bull., 239: 1937.

13. KLEIN,H. et al. Studies on dental caries, i. dental status and dental needs of elementary school-children. Public Health Rep., 53: 751-65, 1938.

14. LAGERLOF, F. Computer simulation of oral fluoride clearance. Comput. Meth. Progr. Blomed., 31: 97-104, 1990.

15. MANDEL, I. D. Dental caries. Am. Sci., 67: 680-8, 1979.

16. NEWBRUN, E. Dental carie in the future: a global view. Proc.Finn.Dent. Soc., 88: 155-61, 1992.

17. NIKIFORUK, G. Understanding dental caries. Etiology and mecanisms, basic and clinical aspects. Basel, Karger, 1985. v. 1.

18. ORGANIZAÇÃO MUNDIAL DA SAÚDE. Levantamento epidemiológico básico de saúde bucal: manual de instrução. 3a. ed. São Paulo, 1991.
19. OLIVEIRA, G. S. C. Cliper em rede; versão 5.01. São Paulo, Érica, 1992.

20. PRESTON, J. D. The future of computers in clinical dentistry. J. Can. Dent. Assoc., 58: 289-94, 1992.

21. QUIVEY, JR., R.G. A computer program designed for the analysis of data from rat caries studies. Caries Res., 25: 1916,1991 .

22. REDE CEDROS. O que fazer nos municípios. Rio de Janeiro, 1992. (Caderno de Saúde Bucal,1).

23. ROSA, A.G.F.et al. Condição de saúde bucal em pessoas de 60 anos ou mais no Município de São Paulo. Rev. Saúde Pública., 26: 155-60, 1992.

24. ROSA, A. G. F. Características epidemiológicas da cárie dental na dentição permanente de escolares do grupo etário de 7 a 14 anos do Estado de São Paulo. São Paulo, 1987. [Tese de Doutoramento - Faculdade de Saúde Pública da USP].

25. ROSA, A. G. F. Saúde bucal na terceira idade. $R G O$, Porto Alegre 41: 97-102, 1993.

26. SALIBA, N. A. et al. Estado de saúde oral da cidade de General Salgado. Estomatol. Cult., 6: 73-7, 1972.

27. SALIBA, N. A. et al. Estado de saúde oral dos escolares da cidade de Valparaíso. Estomatol. Cult., 9: 87-91, 1975.

28. SALIBA, N. A. et al. Prevalência da cárie dentária em escolares da cidade de Birigui. Estomatol. Cult., 9: 277-81, 1975.

29. SALIBA, N. A. et al. Prevalência da cárie dentária em escolares da cidade de Penápolis, Estado de S. Paulo. RGO, Porto Alegre 28: 287-9, 1980.

30. SALIBA, N. A. et al. Contribuição ao estudo sobre a eficiência da aplicação tópica, utilizando-se uma solução acidulada de fluor fosfato. Estomatol. Cult., 9: 101-8, 1975.

31. SEIXAS, J. C. Prefácio. In: Pinto, V. G. Saúde bucal: panorama internacional. Brasília, Ministério da SaúdeSecretaria Nacional de Programas Especiais de Saúde, Divisão Nacional de Saúde Bucal, 1990.

32. STHEEMAN, P. F. Expert systems in dentistry: past performance - future prospects. J. Dent., 20: 68-73, 1992.

33. STRALEY, S. J. Programando em Clipper 5.01. Rio de Janeiro, Ebras Ed., 1991. 2 v.

34. WHITE, S. C. Computer-aided differencial diagnosis of oral radiografic lesions. Dentomaxillofac. Radiol.,18: 53, 1989. 